\title{
Reprodutibilidade na classificação do teste de cristalização do filme lacrimal em pacientes com síndrome de Sjögren
}

\author{
Reproducibility of the classification of ocular ferning patterns in \\ Sjogren's syndrome patients
}

\author{
Sergio Felberg' 1 \\ Hamilcar Cordeiro ${ }^{2}$ \\ Elcio Hideo Sato ${ }^{3}$ \\ Dino Martini Filho ${ }^{4}$ \\ Maria Cristina NIshiwaki-Dantas ${ }^{5}$ \\ Roberto Mitiaki Endo ${ }^{6}$ \\ Paulo Elias Correa Dantas ${ }^{7}$
}

Trabalho realizado no Departamento de Oftalmologia da Santa Casa de Misericórdia de São Paulo (SP) Brasil.

Assistente do Setor de Córnea e Doenças Externas no Departamento de Oftalmologia da Santa Casa de Misericórdia de São Paulo - São Paulo (SP) - Brasil. Pósgraduando nível Doutorado na Universidade Federal de São Paulo - UNIFESP - São Paulo (SP) - Brasil.

Residente do $3^{\circ}$ ano no Departamento de Oftalmologia da Santa Casa de Misericórdia de São Paulo - São Paulo (SP) - Brasil.

Professor da Pós-graduação UNIFESP - São Paulo (SP) - Brasil.

${ }^{4}$ Diretor do Departamento de Anatomia Patológica da Santa Casa de Misericórdia de São Paulo - São Paulo (SP) - Brasil.

${ }^{5}$ Diretora do Departamento de Oftalmologia da Santa Casa de Misericórdia de São Paulo - São Paulo (SP) Brasil.

${ }^{6}$ Professor Assistente no Departamento de Oftalmologia da Santa Casa de Misericórdia de São Paulo - São Paulo (SP) - Brasil.

Chefe do Setor de Córnea e Doenças Externas no Departamento de Oftalmologia da Santa Casa de Misericórdia de São Paulo - São Paulo (SP) - Brasil.

Endereço para correspondência: Departamento de Oftalmologia da Santa Casa de São Paulo. Sergio Felberg. R. Cesário Mota Júnior, 112 - São Paulo (SP) CEP 01221-900

E-mail: sergiofelberg@oftalmosantacasa.com.br

Recebido para publicação em 31.05.2006

Última versão recebida em 24.10.2007

Aprovação em 22.11.2007

\begin{tabular}{|l|}
\hline RESUMO \\
\hline Objetivo: Verificar a reprodutibilidade da classificação dos padrões do \\
teste de cristalização do filme lacrimal utilizando cinco examinadores \\
diferentes e comparar os padrões de cristalização de pacientes portadores \\
da síndrome de Sjögren com os de indivíduos não portadores de doenças \\
da superfície ocular. Métodos: Análise da cristalização da lágrima de 29 \\
pacientes com Sjögren e 45 pacientes sem doenças da superfície ocular, \\
através de microscópio com luz polarizada, utilizando a classificação de \\
Rolando. Para fins estatísticos foi estudada a curva ROC (Receiver Ope- \\
rating Characteristic) para determinar a melhor nota de corte do exame que \\
separa indivíduos normais dos portadores da síndrome, índice de concor- \\
dância Kappa (p<0,0001) para averiguar a reprodutibilidade da classifica- \\
ção dos padrões de cristalização e tabelas de contingência para compara- \\
ção dos resultados entre os grupos. Resultados: Com os padrões agrega- \\
dos (I com II e III com IV) a concordância dos examinadores foi alta \\
(Kappa=0,82 a0,97 ep<0,0001). Por meio da curva ROC obtivemos nota de \\
corte de 2,50 para o diagnóstico de Sjögren. Na comparação entre os \\
grupos, o grupo normal recebeu predominantemente classificações de \\
padrões I e II, ao passo que o grupo com Sjögren, padrões III e IV. \\
Conclusões: Houve concordância com a literatura quanto aos padrões \\
encontrados nos pacientes com Sjögren e nos pacientes sem doença da \\
superfície ocular. A classificação do teste de cristalização é reprodutível \\
quando utilizada a classificação de Rolando. \\
\hline
\end{tabular}

Descritores: Síndrome de Sjogren/diagnóstico; Cristalização/métodos; Cristalização/classificação; Lágrimas/química; Técnicas de diagnóstico oftalmológico; Reprodutibilidade dos testes

\section{INTRODUÇÃO}

A síndrome de Sjögren (SS) é doença sistêmica, de provável etiologia autoimune e que apresenta evolução crônica, sendo caracterizada anatomopatologicamente pela infiltração linfo-plasmocitária das glândulas exócrinas, principalmente as lacrimais e as salivares ${ }^{(1)}$. A doença causa inflamação tecidual com progressiva substituição dos ácinos por tecido fibroso, levando às disfunções dessas glândulas e originando os característicos quadros de xeroftalmia e xerostomia encontrados nesses pacientes. Na forma primária da SS, o comprometimento das glândulas exócrinas ocorre isoladamente e na forma secundária, acompanhado de outra doença autoimune, sendo a artrite reumatóide e o lúpus eritematoso sistêmico as mais freqüentes ${ }^{(2)}$. 
Olho seco é a principal manifestação ocular da síndrome e ocorre por diminuição progressiva do componente aquoso do filme lacrimal, em função da destruição das glândulas lacrimais principais e acessórias $^{(3-4)}$. Sintomas oculares, como sensação de corpo estranho, queimação, prurido e vermelhidão são comuns já nas fases iniciais da doença ${ }^{(5)}$. Em consequiência da falta de lubrificação, as córneas dos pacientes com SS estão predispostas ao desenvolvimento de defeitos epiteliais crônicos, vascularização, úlceras estéreis ou infectadas. Perfuração ocular pode ocorrer nos casos mais graves, podendo haver perda da integridade ocular e cegueira irreversível ${ }^{(6-7)}$.

Dentre os testes utilizados na avaliação oftalmológica dos pacientes com olho seco, os mais utilizados são: teste de Schirmer, tempo de rotura do filme lacrimal e a coloração da superfície ocular com corantes (rosa bengala e fluoresceína) ${ }^{(8-10)}$. No entanto, outras provas menos usuais na prática clínica também estão disponíveis para a avaliação de pacientes com suspeita ou diagnóstico estabelecido de olho seco, como citologia conjuntival de impressão, análise do perfil protéico do filme lacrimal, medida da osmolaridade da lágrima e o teste de cristalização do filme lacrimal, dentre outros ${ }^{(11-14)}$.

O primeiro relato a respeito do estudo de cristais obtidos em amostras de lágrima humana foi publicado por cientistas franceses em 1791. No entanto, somente dois séculos depois, após as publicações de Tabbara, de Rolando e de Norm, esta modalidade de avaliação do filme lacrimal passou a ser mais frequientemente encontrada nos artigos científicos ${ }^{(15-18)}$. O exame se baseia no princípio de que a lágrima, quando seca, apresenta cristalização de alguns de seus componentes, que assumem formatos variados, lembrando folhas de samambaia.

Rolando classificou os achados em quatro grupos distintos, de acordo com a presença e exuberância destas folhas e posteriormente, ao estudar um grupo de pacientes com olho seco de várias etiologias, percebeu que os padrões encontrados eram diferentes daqueles obtidos nas lágrimas de indivíduos normais ${ }^{(16)}$.

Não há consenso entre os pesquisadores a respeito da natureza destes mosaicos formados no teste de cristalização. Alguns acreditam tratar-se principalmente de cloreto de sódio ${ }^{(19)}$. Outros afirmam que os depósitos correspondem à mucina da lágrima, mais especificamente glicoproteínas ${ }^{(19-21)}$. Estudo in vitro demonstrou que glicoproteínas semelhantes às encontradas no filme lacrimal, quando dissolvidas em água destilada, não formam os clássicos padrões em folha de samambaia. Outras variáveis, portanto, colaboram para seu aparecimento ${ }^{(17)}$.

Inúmeros estudos publicados nos últimos anos utilizaram o teste de cristalização do filme lacrimal como ferramenta na avaliação de pacientes portadores de doenças oculares diversas, como ceraconjuntivite seca, conjuntivite folicular crônica, intolerância ao uso de lentes de contato, síndrome de Stevens-Johnson, penfigóide ocular cicatricial, avitaminoses, dentre outras. Todos utilizaram o modelo proposto por Rolando para classificar seus achados ${ }^{(15,22-23)}$.

Considerando que a classificação proposta por Rolando para avaliação dos padrões encontrados no teste de cristalização do filme lacrimal apresenta certo grau de subjetividade uma vez que as diretrizes propostas pelo autor para definir cada um dos quatro padrões não são exatas ou mensuráveis; que a classificação atribuída a um mesmo achado no teste de cristalização pode variar entre observadores, quando utilizado o modelo de classificação proposto por Rolando e finalmente que não foi encontrado trabalho disponível na literatura que tenha estudado a reprodutibilidade na classificação dos achados no teste de cristalização do filme lacrimal utilizando o modelo de classificação proposto por Rolando, este trabalho teve como objetivos verificar a reprodutibilidade na classificação dos padrões de cristalização do filme lacrimal das amostras de lágrima obtidas de 65 pacientes, examinadas por cinco observadores diferentes e comparar os padrões encontrados no teste de cristalização do filme lacrimal de pacientes portadores da síndrome de Sjögren com os de indivíduos não portadores de doenças da superfície ocular.

\section{MÉTODOS}

Este trabalho foi realizado no Departamento de Oftalmologia da Santa Casa de São Paulo, (Ambulatório de Superfície Ocular e Lágrima) e no Departamento de Patologia da Santa Casa de São Paulo.

Trata-se de estudo prospectivo, mascarado, agregado, observacional, analítico e transversal.

\section{Pacientes}

Foram constituídos dois grupos de pacientes para este estudo: Grupo A e Grupo B.

Grupo A - Vinte e nove pacientes que obedeceram aos seguintes critérios:

\section{Critérios de inclusão}

Serem pacientes cadastrados no Ambulatório de Superfície Ocular e Lágrima (S.O.L.) da Seção de Córnea e Doenças Externas do Departamento de Oftalmologia da Santa Casa de São Paulo, portadores da síndrome de Sjögren. Os critérios utilizados para caracterizar os pacientes como portadores da síndrome foi o adotado pelo Consenso Americano-Europeu ${ }^{(24)}$, terem maioridade legal, serem alfabetizados, terem lido e assinado o Termo de Consentimento Livre após esclarecimento, fornecido pelos pesquisadores e finalmente estarem há pelo menos duas semanas sem uso de medicação ocular tópica.

\section{Critérios de exclusão}

Apresentarem doença palpebral ou ocular não atribuída à síndrome de Sjögren, serem usuários de lentes de contato, possuírem oclusão temporária ou definitiva dos pontos lacrimais, estarem em período de gestação ou amamentação, estarem utilizando uma ou mais drogas das classes dos anti-depressivos, anti-histamínicos, neurolépticos ou hipotensores diuréticos.

Grupo B - Quarenta e cinco pacientes que compareceram ao Pronto Atendimento de Oftalmologia da Santa Casa de São 
Paulo para exame de refração e que obedeceram aos seguintes critérios.

\section{Critérios de inclusão}

Não apresentarem queixas de sensação de olho seco, irritação ocular, queimação ocular e sensação de corpo estranho, não terem sido constatadas nestes pacientes doenças da superfície ocular após exame biomicroscópico de ambos os olhos, apresentarem valores acima de $15 \mathrm{~mm}$ no teste de Schirmer-I (realizado sem utilização de anestésico e com leitura realizada após cinco minutos), apresentarem pontuação menor ou igual a quatro na escala de Van Bijesterveld ${ }^{(8)}$ no exame de coloração da superfície ocular com colírio de Rosa Bengala 1\%, estarem há pelo menos duas semanas sem uso de medicação ocular tópica, terem maioridade legal, serem alfabetizados e terem lido e assinado o Termo de Consentimento Livre após esclarecimento fornecido pelos pesquisadores.

\section{Critérios de exclusão}

Os mesmos do Grupo A.

\section{O teste de cristalização da lágrima}

Todas as amostras de lágrima foram colhidas na mesma sala do Departamento de Oftalmologia da Santa Casa de São Paulo, com portas e janelas fechadas. Nos horários das coletas, temperatura e umidade relativa do ar dentro desta sala foram registradas com aparelho termo-higrômetro. Nos períodos de coleta, a temperatura máxima da sala variou entre 28,7 e $26,3^{\circ} \mathrm{C}$ e a temperatura mínima variou entre 22,6 e $19,8^{\circ} \mathrm{C}$. A umidade relativa do ar variou entre 69 e $48 \%$.

Os pacientes foram acomodados na lâmpada de fenda e, com tubo de vidro capilar, amostra de quantidade variável da lágrima foi coletada do fundo de saco do olho direito de todos os pacientes.

Em seguida, foi depositada na superfície de uma lâmina de vidro limpa uma gota com diâmetro que variou entre dois e três milímetros, no centro de um círculo marcado previamente com caneta azul ou vermelha. A lágrima foi deixada secar em temperatura ambiente por cerca de dez minutos antes de ser realizada a sua leitura. Todas as coletas de lágrima foram realizadas pelo mesmo examinador. A leitura das lâminas foi feita com auxílio de microscópio de luz polarizada. Foram obtidas fotografias digitais do local correspondente à gota de lágrima seca de cada paciente, com câmera acoplada ao microscópio (Sony Cybershot), em aumentos de 5, 10, 20 e 40 vezes e resolução de 3 megapixels. As fotografias foram reveladas em papel retangular, com tamanho de $10 \mathrm{~cm}$ (altura) por $15 \mathrm{~cm}$ (largura) e examinadas por cinco pesquisadores, separadamente. Todos os observadores analisaram todas as fotos e desconheciam o grupo ao qual o paciente responsável pela fotografia examinada pertencia (se grupo A ou B). Dos examinadores, alguns apresentavam experiência prévia variável com o teste, outros nenhuma experiência.

Para a classificação, os achados do teste de cristalização do filme lacrimal foram divididos em quatro padrões, de acordo com o modelo proposto por Rolando ${ }^{(16)}$. De acordo com este autor, o padrão I é aquele cuja cristalização da lágrima origina árvores de cristais multi-ramificados, sem espaços entre vazios entre as arborificações (Figura 1). No padrão II os ramos são mais curtos e as arborificações são menores, com mais espaços entre elas (Figura 2). No padrão III os espaços entre as arborificações é amplo e as ramificaçoes são raras (Figura 3) e finalmente o padrão IV é caracterizado por grumos de cristais que raramente formam pequenas arborificações (Figura 4).

Todas as anotações com relação aos resultados obtidos foram arquivadas em fichários e submetidas posteriormente à análise estatística.

\section{Análise estatística}

- Os escores dos cinco examinadores foram analisados descritivamente pelas freqüências simples. A correlação entre as classificações atribuídas aos mesmos pacientes pelos cinco examinadores diferentes foi obtida com coeficiente de concordância Kappa, que assumiu valores entre -1 (completa discordância) e +1 (completa concordância);

- para a interpretação dos valores de Kappa, utilizou-se a caracterização em faixas de valores para os graus de concordância, que correlacionam valores acima de 0,75 a uma concordância excelente, valores abaixo de 0,40 a uma concordância pobre e valores entre 0,40 e 0,75 a boa concordância;

- curva ROC (Receiver Operating Characteristic) foi utilizada para a determinação do melhor "ponto de corte" que define o paciente como sendo portador ou não da síndrome de Sjögren;

- para significância estatística considerou-se $p$ menor que cinco por cento.

Este estudo teve seu protocolo aprovado pelo Comitê de Ética e Pesquisa da Santa Casa de São Paulo, com o número 16203.

\section{RESULTADOS}

Dentre os 29 pacientes que compunham o grupo A (síndrome de Sjögren), 26 (90\%) pertenciam ao sexo feminino. A idade dos pacientes do grupo variou entre 27 e 71 anos (média

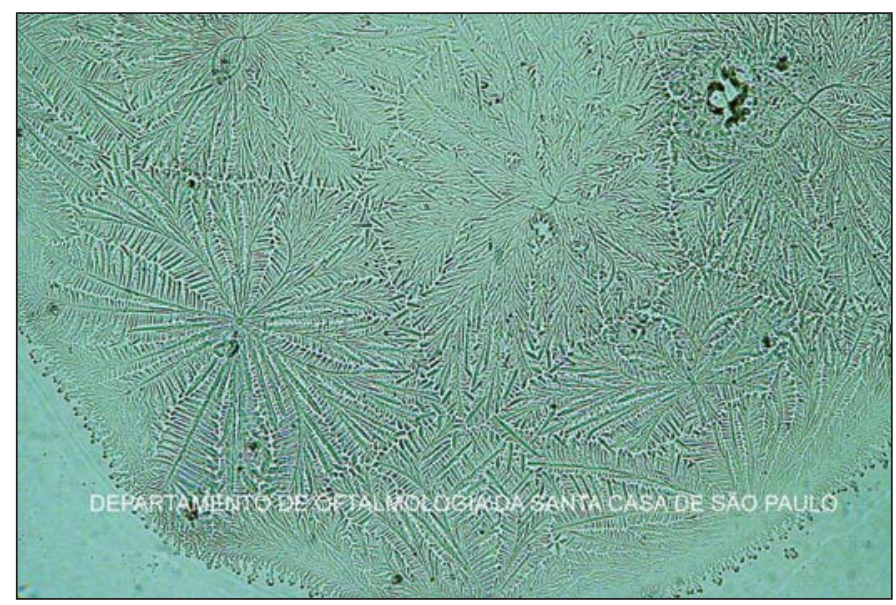

Figura 1 - Padrão de cristalização tipo I. Fotografia em aumento de 10x - microscopia de luz polarizada. 


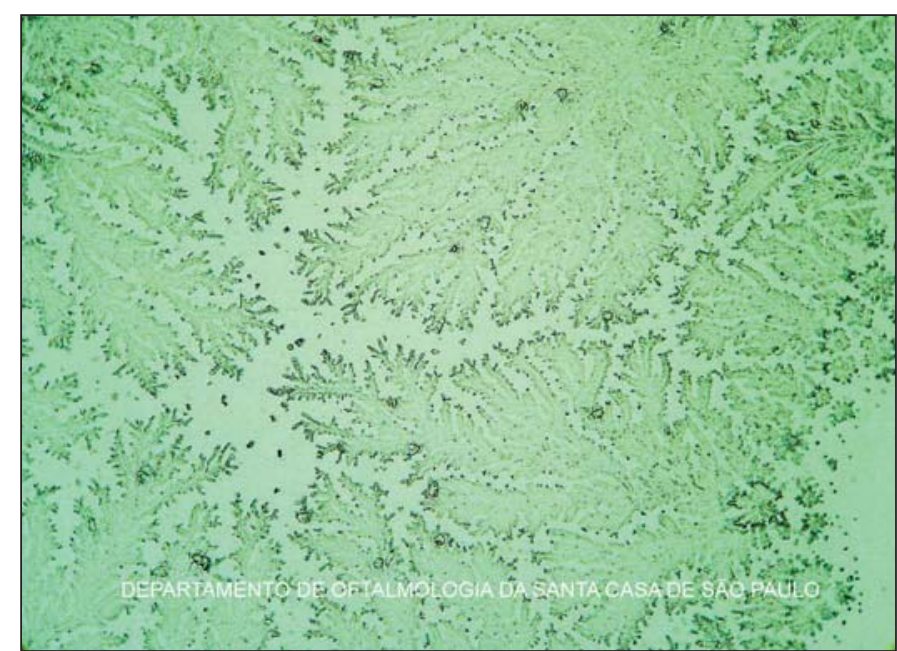

Figura 2 - Padrão de cristalização tipo II. Fotografia em aumento de 10x - microscopia de luz polarizada.

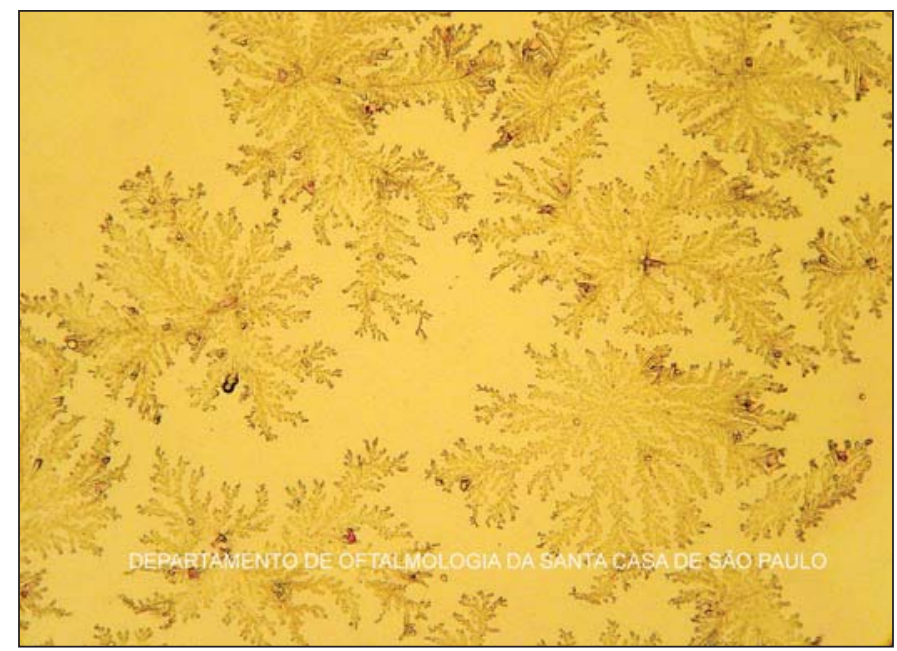

Figura 3 - Padrão de cristalização tipo III. Fotografia em aumento de 10x - microscopia de luz polarizada.

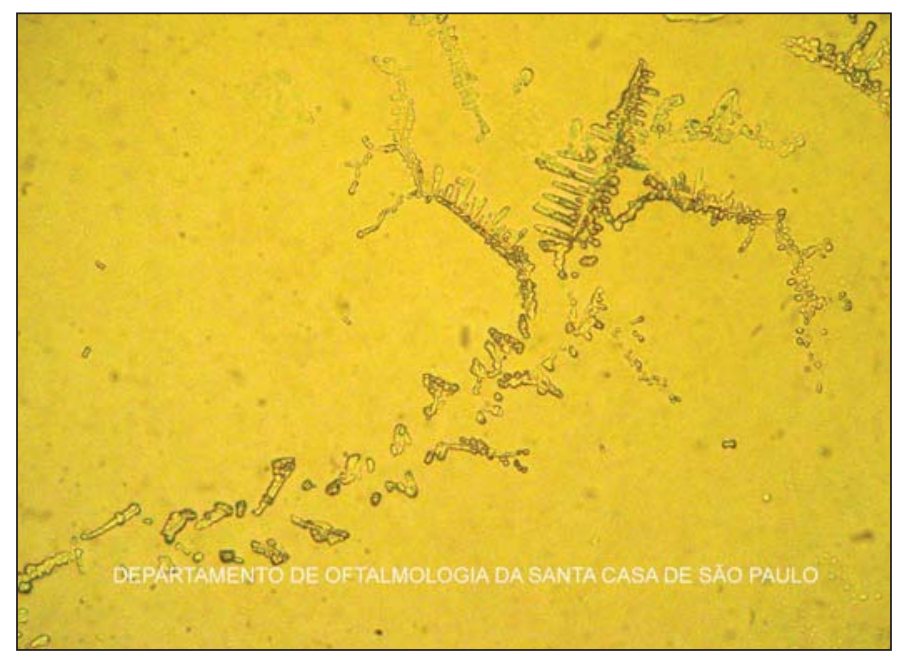

Figura 4 - Padrão de cristalização tipo IV. Fotografia em aumento de $10 x$ - microscopia de luz polarizada. de 52,65 $\pm 9,8)$. Dos 45 pacientes que compunham o grupo B (controle), $22(48,9 \%)$ pertenciam ao sexo feminino e 23 $(51,1 \%)$ ao masculino. A idade variou entre 19 e 72 anos (média de $43,57 \pm 15,42)$.

A tabela 1 apresenta as estimativas para os coeficientes Kappa para cada par de examinadores com os escores analisados separadamente (I, II, III e IV), evidenciando correlação moderada entre as análises de cada um dos padrões quando comparadas entre cada par de examinadores.

A tabela 2 apresenta as estimativas para os coeficientes Kappa para cada par de examinadores com os escores agregados I com II e III com IV. A correlação foi alta para separar os padrões I e II dos padrões III e IV.

A tabela 3 apresenta os valores da curva ROC para cada observador.

Optou-se por números fracionados na análise da curva pois tratando-se de escores, as frações foram capazes de separar os escores entre si. Assim, o número 1,5 separa o escore I do escore II, por exemplo. De acordo com esta tabela, o ponto que melhor separou pacientes normais dos portadores da síndrome foi 2,5, isto é, o padrão II do padrão III.

\section{DISCUSSÃO}

Embora existam diversas publicações utilizando o teste de cristalização na avaliação do filme lacrimal de pacientes com doenças da superfície ocular, nenhum trabalho havia estudado a reprodutibilidade na classificação dos achados. Como a decisão pela escolha de cada escore do padrão do teste de cristalização (I, II, III e IV) envolve certa subjetividade, este trabalho teve a intenção de estudar, independentemente da experiência prévia de cada examinador com o teste, a capacidade de classificarem os mesmos achados, de forma idêntica ou semelhante. Com relação à reprodutibilidade na classificação dos padrões encontrados no teste, notamos, ao observar a tabela 1, correlação abaixo da considerada ideal quando os

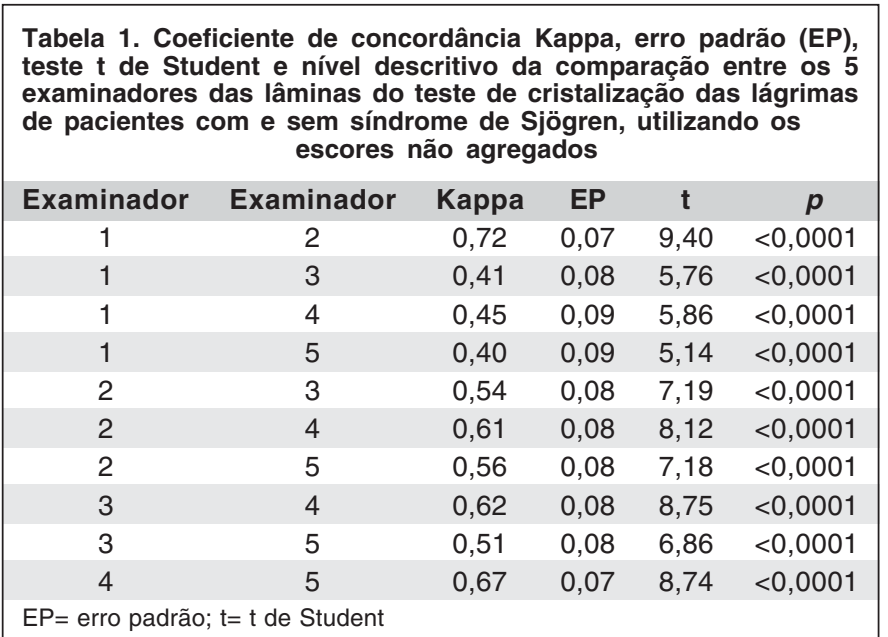


diferentes escores são analisados um a um para cada paciente e comparados entre todos os examinadores. Entre os examinadores 1 e 3 a correlação foi a mais baixa e entre os examinadores 1 e 2, a mais alta. A experiência variável com o teste de cristalização e a interpretação pessoal do gabarito proposto por Rolando para definir cada padrão da classificação pode explicar a baixa correlação entre os padrões encontrados. A maior discordância foi encontrada entre as classificações I e II. Porém, quando esses dois valores foram agregados e separados dos padrões III e IV, a concordância de classificação entre os cinco examinadores foi alta para ambos os grupos

\begin{tabular}{|c|ccccc|}
\hline $\begin{array}{l}\text { Tabela 2. Coeficiente de concordância Kappa, erro padrão (EP), } \\
\text { teste t de Student e nível descritivo da comparação entre os 5 } \\
\text { examinadores das lâminas do teste de cristalização das lágrimas } \\
\text { de pacientes com e sem síndrome de Sjögren, utilizando os escores } \\
\text { agregados (I com II e III com IV) } \\
\text { Examinador } \\
\text { Examinador }\end{array}$ Kappa & EP & t & p \\
\hline 1 & 2 & 0,92 & 0,05 & 7,47 & $<0,0001$ \\
1 & 3 & 0,82 & 0,08 & 6,71 & $<0,0001$ \\
1 & 4 & 0,85 & 0,07 & 6,95 & $<0,0001$ \\
1 & 5 & 0,92 & 0,05 & 7,47 & $<0,0001$ \\
2 & 3 & 0,82 & 0,08 & 6,67 & $<0,0001$ \\
2 & 4 & 0,86 & 0,07 & 6,92 & $<0,0001$ \\
2 & 5 & 0,93 & 0,05 & 7,46 & $<0,0001$ \\
3 & 4 & 0,97 & 0,03 & 7,79 & $<0,0001$ \\
3 & 5 & 0,89 & 0,06 & 7,24 & $<0,0001$ \\
4 & 5 & 0,93 & 0,05 & 7,50 & $<0,0001$ \\
EP= erro-padrão; $t=t$ te Student & & & & \\
\hline
\end{tabular}

(Tabela 2). A justificativa dada por Rolando para considerar determinado padrão como sendo I ou II pode não estar clara para os examinadores e sua redefinição poderá facilitar a individualização de cada um destes padrões pelos pesquisadores, principalmente valorizando a ausência de espaços entre os "fernings" no padrão I e a presença deles no padrão II.

Quando são comparados os padrões encontrados no teste entre pacientes normais e com síndrome de Sjögren, com exceção de um, todos os pacientes que receberam classificação I de todos os examinadores pertenciam ao grupo dos indivíduos normais enquanto os que receberam classificação IV pertenciam ao grupo dos portadores da síndrome.

Entretanto, os escores II e III foram atribuídos aos pacientes de ambos os grupos, embora uma freqüência maior do escore II tenha sido evidenciada no grupo dos normais e do escore III no grupo dos doentes. Esse "corte" entre os padrões II e III obtido com a curva ROC (Tabela 3) representa o ponto ótimo entre sensibilidade e especificidade do teste e coincide com o sugerido por Rolando quando este analisou o comportamento do teste em pacientes com ceratoconjuntivite seca, sem especificar os sub-tipos da doença.

Alguns autores estudaram a reprodutibilidade do teste de cristalização utilizando a classificação proposta por Rolando num grupo de pacientes portadores de olho seco de etilogia variada e encontraram excelente concordância na classificação entre observadores diferentes ao analisarem separadamente as lâminas obtidas com amostras de lágrimas do grupo de estudo $(80,62 \%)^{(25)}$.

\begin{tabular}{|c|c|c|c|c|}
\hline & Ponto de Corte & Sensibilidade & Especificidade & 1 - Especificidade \\
\hline Examinador 1 & $\begin{array}{l}0,00 \\
1,50 \\
2,50 \\
3,50 \\
5,00\end{array}$ & $\begin{array}{l}1,00 \\
1,00 \\
\mathbf{0 , 8 5} \\
0,50 \\
0,00\end{array}$ & $\begin{array}{l}0,00 \\
0,64 \\
1,00 \\
1,00 \\
1,00\end{array}$ & $\begin{array}{l}1,00 \\
0,36 \\
\mathbf{0 , 0 0} \\
0,00 \\
0,00\end{array}$ \\
\hline Examinador 2 & $\begin{array}{l}0,00 \\
1,50 \\
2,50 \\
3,50 \\
5,00\end{array}$ & $\begin{array}{l}1,00 \\
1,00 \\
0,90 \\
0,65 \\
0,00\end{array}$ & $\begin{array}{l}0,00 \\
0,47 \\
0,98 \\
1,00 \\
1,00\end{array}$ & $\begin{array}{l}1,00 \\
0,53 \\
0,02 \\
0,00 \\
0,00\end{array}$ \\
\hline Examinador 3 & $\begin{array}{l}0,00 \\
1,50 \\
2,50 \\
3,50 \\
5,00\end{array}$ & $\begin{array}{l}1,00 \\
1,00 \\
0,95 \\
0,70 \\
0,00\end{array}$ & $\begin{array}{l}0,00 \\
0,31 \\
\mathbf{0 , 9 3} \\
1,00 \\
1,00\end{array}$ & $\begin{array}{l}1,00 \\
0,69 \\
0,07 \\
0,00 \\
0,00\end{array}$ \\
\hline Examinador 4 & $\begin{array}{l}0,00 \\
1,50 \\
2,50 \\
3,50 \\
5,00\end{array}$ & $\begin{array}{l}1,00 \\
0,95 \\
0,95 \\
0,65 \\
0,00\end{array}$ & $\begin{array}{l}0,00 \\
0,56 \\
0,96 \\
1,00 \\
1,00\end{array}$ & $\begin{array}{l}1,00 \\
0,44 \\
0,04 \\
0,00 \\
0,00\end{array}$ \\
\hline Examinador 5 & $\begin{array}{l}0,00 \\
1,50 \\
2,50 \\
3,50 \\
5,00\end{array}$ & $\begin{array}{l}1,00 \\
1,00 \\
0,95 \\
0,75 \\
0,00\end{array}$ & $\begin{array}{l}0,00 \\
0,56 \\
1,00 \\
1,00 \\
1,00\end{array}$ & $\begin{array}{l}1,00 \\
0,44 \\
0,00 \\
0,00 \\
0,00\end{array}$ \\
\hline
\end{tabular}




\section{CONCLUSÃO}

Não houve, durante todo período de estudo, acidentes relacionados à técnica de coleta da lágrima para o exame de cristalização. Não há na literatura trabalho que relate acidentes durante a coleta de amostras da lágrima com tubo capilar ou outras complicações associadas ao método.

Como a reprodutibilidade na classificação dos padrões de cristalização do filme lacrimal, segundo o modelo de classificação proposto por Rolando, foi boa quando as amostras de lágrimas foram examinadas uma a uma para cada um dos padrões, porém, demonstrou-se excelente quando agrupados os padrões I com II e III com IV, o presente estudo demonstrou que o teste de cristalização do filme lacrimal pode ter grande utilidade na avaliação dos suspeitos de terem a síndrome de Sjögren e que, devido à sua facilidade de realização, baixo custo, baixo risco de complicações e boa reprodutibilidade na classificação dos achados, pode fazer parte da prática clínica diária dos oftalmologistas que avaliam estes pacientes.

\section{ABSTRACT}

Purpose: To verify the reproducibility of Rolando's classification of the tear ferning test using five different examiners and to compare the patterns of crystallization found in Sjögren's syndrome patients and normal subjects. Methods: Tear ferning analysis of 29 patients with Sjögren's syndrome and of 45 patients without ocular disease were done using polarized light microscopy and the Rolando classification for tear ferning. Five examiners classified the ferning patterns of all the patients. ROC curve (Receiver Operating Characteristic) was used to find out the best score for the correct syndrome diagnosis. Kappa index $(\mathrm{p}<0.0001)$ was used to compare the results of the examiners among them and check the test's reproducibility. Charts were drawn to compare the two groups' results. Results: Throught the ROC curve the score of 2.50 for diagnosis of Sjögren's syndrome was stabilished. Considering the aggregated patterns I with II and III with IV, the examinors' level of pattern agreement was excellent (Kappa ranging from 0.82 to $0.97, \mathrm{p}<0.0001$ ). The group with Sjögren's syndrome was classified mostly as patterns III and IV and the patients without ocular disease mostly as I and II. Conclusion: The patterns associated with Sjögren's syndrome and normal patients matched the ones in the literature. The tear ferning test classification is reproductible when the Rolando classification was used for Sjögren's syndrome patients.

Keywords: Sjögren's syndrome/diagnosis; Crystallization/methods; Crystallization/classification; Tears/chemistry; Diagnostic techniques, ophthalmological; Reproducibility of results

\section{REFERÊNCIAS}

1. Bowman SJ, Pillemer S, Jonsson R, Asmussen K, Vitali C, Manthorpe R, Sutcliffe N. Contributors to and participants at the workshop. Revisiting Sjogren's syndrome in the new millennium: perspectives on assessment and outcome measures. Report of a workshop held on 23 March 2000 at Oxford, UK. Rheumatology (Oxford). 2001;40(10):1180-8.

2. Asmussen KH, Bowman SJ. Outcome measures in Sjogren's syndrome. Rheumatology (Oxford). 2001;40(10):1085-8.

3. Felberg S, Sato EH, Dantas PE. Síndrome de Sjögren: Análise e comparação dos diferentes critérios diagnósticos. Vision Pan-America. 2005;4(4):14-6.

4. Daniels TE, Whitcher JP. Association of patterns of labial salivary gland inflammation with keratoconjunctivitis sicca. Analysis of 618 patients with suspected Sjogren's syndrome. Arthritis Rheum. 1994;37(6):869-77. Comment in: Arthritis Rheum. 1995;38(6):869-72.

5. Jonsson R, Moen K, Vestrheim D, Szodoray P. Current issues in Sjogren's syndrome. Oral Dis. 2002;8(3):130-40.

6. Rehman Hu. Sjogren's syndrome. Yonsei Med J. 2003;44(6):947-54

7. Felberg S, Dantas PEC. Atualização no diagnóstico e tratamento da síndrome de Sjögren. Arq Bras Oftalmol. 2006;69(6):959-63.

8. Van Bijsterveld OP. Diagnostic tests in the Sicca syndrome. Arch Ophthalmol. 1969;82(1):10-4.

9. Fox RI, Howell FV, Bone RC, Michelson P. Primary Sjogren syndrome: clinical and immunopathologic features. Semin Arthritis Rheum. 1984;14(2):77-105.

10. Felberg S, Sato EH. O componente aquoso do filme lacrimal. In: Sousa, LB, Freitas D, Vieira L. Doenças imunológicas da conjuntiva e glândula lacrimal. São Paulo: Phoenix; 2005. p.

11. Bron AJ, Tiffany JM, Yokoi N, Gouveia SM. Using osmolarity to diagnose dry eye: a compartmental hypothesis and review of our assumptions. Adv Exp Med Biol. 2002;506(Pt B):1087-95.

12. Jackson JA, Perrigin JA. Relationship of impression cytology and tear ferning to reports of dry eye. J Am Optom Assoc. 1999;70(3):187-92.

13. Kruize AA, van Bijsterveld OP, Hené RJ, de Wilde PC, Feltkamp TE, Kater $\mathrm{L}$, et al. Long-term course of tear gland function in patients with keratoconjunctivitis sicca and Sjogren's syndrome. Br J Ophthalmol. 1997;81(6): 435-8. Comment in: Br J Ophthalmol. 1997;81(6):426.

14. Versura P, Frigato M, Bernabini B, Mule R, Malavolta N, Campos EC. [Ocular surface analysis in patients affected with rheumatic diseases]. Reumatismo. 2004;56(4):262-71. Italian.

15. Tabbara KF, Okumoto M. Ocular ferning test. A qualitative test for mucus deficiency. Ophthalmology. 1982;89(6):712-4.

16. Rolando M. Tear mucus ferning test in normal and keratoconjuntivitis sicca eyes. Chibret Int J Ophthamol. 1984;2(4):32-41.

17. Rolando M. The fern test; a critical analysis. Obstet Gynecol. 1958;11(1):30-4.

18. Norn M. Ferning in conjunctival-cytologic preparations. crystallization in stained semiquantitative pipette samples of conjunctival fluid. Acta Ophthalmol (Copenh).1987;65(1):118-22.

19. Liotet S, Kogbe O, Schemann JF. [Crystallization of tears: a test of the quality of the lacrimal film?] Bull Soc Ophtalmol Fr. 1987;87(3):321-4.

20. Rolando M, Baldi F, Calabria GA. Tear mucus ferning test in keratoconjunctivitis sicca. In: Holly FJ, editor. The preocular tear film in health disease, and contact lens wear. Lubbock, Texas; Dry Eye Institute; 1986. p.203-10.

21. Wyon NM, Wyon DP. Measurement of acute response to draught in the eye. Acta Ophthalmol (Copenh). 1987;65(4):385-92.

22.Rolando M, Baldi F, Calabria G. Tear mucus crystallization in children with cystic fibrosis. Ophthalmologica. 1988;197(4):202-6.

23. Kogbe O, Liotet S. An interesting use of the study of tear ferning patterns in contactology. Ophthalmologica. 1987;194(2-3):150-3.

24. Vitali C, Bombardieri S, Jonsson R, Moutsopoulos HM, Alexander EL, Carsons SE, Daniels TE, Fox PC, Fox RI, Kassan SS, Pillemer SR, Talal $\mathrm{N}$, Weisman MH; European Study Group on Classification Criteria for Sjogren's Syndrome. Classification criteria for Sjögren's syndrome: a revised version of the European criteria proposed by the American-European Consensus Group. Ann Rheum Dis 2002;61(6):554-8.

25. Pensyl CD, Dillehay SM. The repeatability of tear mucus ferning grading. Optom Vis Sci. 1998;75(8):600-4. 